University of Nebraska - Lincoln

DigitalCommons@University of Nebraska - Lincoln

Axel Enders Publications

Research Papers in Physics and Astronomy

2010

\title{
Magnetic susceptibility of nanoscale Kondo systems
}

\author{
Ralph Skomski \\ University of Nebraska-Lincoln, rskomski2@unl.edu \\ R. Zhang \\ University of Nebraska, Lincoln \\ Parashu Kharel \\ University of Nebraska-Lincoln, pkharel2@unl.edu \\ Axel Enders \\ University of Nebraska-Lincoln, a.enders@me.com \\ Sy_Hwang Liou \\ University of Nebraska-Lincoln, sliou@unl.edu \\ See next page for additional authors
}

Follow this and additional works at: https://digitalcommons.unl.edu/physicsenders

Part of the Physics Commons

Skomski, Ralph; Zhang, R.; Kharel, Parashu; Enders, Axel; Liou, Sy_Hwang; and Sellmyer, D. J., "Magnetic susceptibility of nanoscale Kondo systems" (2010). Axel Enders Publications. 25.

https://digitalcommons.unl.edu/physicsenders/25

This Article is brought to you for free and open access by the Research Papers in Physics and Astronomy at DigitalCommons@University of Nebraska - Lincoln. It has been accepted for inclusion in Axel Enders Publications by an authorized administrator of DigitalCommons@University of Nebraska - Lincoln. 


\section{Authors}

Ralph Skomski, R. Zhang, Parashu Kharel, Axel Enders, Sy_Hwang Liou, and D. J. Sellmyer 


\title{
Magnetic susceptibility of nanoscale Kondo systems
}

\author{
R. Skomski, ${ }^{a}$ R. Zhang, P. Kharel, A. Enders, S.-H. Liou, and D. J. Sellmyer \\ Department of Physics and Astronomy and Nebraska Center for Materials and Nanoscience, University of \\ Nebraska, Lincoln, Nebraska 68588, USA
}

(Presented 21 January 2010; received 31 October 2009; accepted 19 November 2009; published online 22 April 2010)

\begin{abstract}
The mesoscopic Kondo effect in metallic nanoparticles containing a magnetic impurity is investigated by model calculations. A Maxwell-Garnett approach is used to approximately determine the resistivity of doped nanoparticles in a matrix, and the magnetic susceptibility is estimated from the confinement of the conduction electrons. Conductivity measurements of nanoparticles embedded in a matrix are difficult to realize, because metallic matrices distort the Kondo cloud, whereas insulating or semiconducting matrices yield a very weak signal. By comparison, susceptibility measurements do not suffer from these shortcomings. The Kondo effect survives in nanoparticles even if the cluster size is much smaller than the Kondo screening cloud, but the effective Curie constant becomes constant below a particle-size dependent transition temperature and the temperature dependence of the susceptibility is no longer universal.
\end{abstract}

(C) 2010 American Institute of Physics. [doi:10.1063/1.3365134]

\section{INTRODUCTION}

The Kondo effect has recently attracted renewed attention, stimulated by progress in various areas of nanotechnology. ${ }^{1-6}$ In bulk materials, the effect is usually associated with the resistance minimum, which was discovered in 1930 by Meissner and Voigt ${ }^{7}$ and explained by Kondo in $1964 .^{8}$ It is caused by magnetic impurities in the metallic host, and its explanation involves an integration over all $k$-vectors of the host's conduction electrons. This $k$-space integration is the reason for the occurrence of the logarithmic term governing the resistivity, which increases as $\ln (T)$ with decreasing temperature. ${ }^{8}$ However, the large number of $k$-vectors is only one aspect of the phenomenon. From a many-body point of view, the Kondo effect is caused by competing Coulomb and hopping integrals. It involves a well-localized orbital having a low on-site energy $E_{\mathrm{o}}$ for the first electron but where the presence of a second electron of opposite spin is inhibited by a high Coulomb energy. A hopping integral $t$ connects the localized orbital to a single delocalized orbital and yields the system's "single-orbital" Kondo temperature $T_{\mathrm{K}}=2 t^{2} / k_{\mathrm{B}} E_{\mathrm{o}}{ }^{9,10}$ Below this temperature, the impurity spin is antiferromagnetically (AFM) coupled to the delocalized electron, ${ }^{9,10}$ similar to the AFM interaction of magnetic impurities with the Kondo screening cloud in bulk metals.

Nanostructuring makes it possible to control the wavelength of the conduction electrons that contribute to the Kondo effect. Consider, for example, a single magnetic impurity in an approximately spherical metal cluster of radius $R$. The screening cloud, whose size is given by the Kondo coherence length $\xi \sim v_{\mathrm{F}} / T_{\mathrm{K}},{ }^{6}$ can be much larger than typical mesoscopic (nanostructural) feature sizes, and the question arises how nanoscale confinement affects the Kondo effect. In the literature, there are two opposing views. On the one

\footnotetext{
${ }^{a)}$ Electronic mail: rskomski@neb.rr.com.
}

hand, the Kondo effect involves a large number of conduction electrons, and this continuum is essential for the understanding of the resistance minimum. ${ }^{8,11}$ On the other hand, as outlined above, the basic quantum-mechanical feature behind the Kondo effect is the interaction of a well-localized impurity spin with delocalized electrons, and this coupling is already realized for a single delocalized or conduction electron. ${ }^{9,10}$ This means that basic features of the Kondo effect survive even for very few electrons. An extreme view is that nanostructuring has little or no effect on the Kondo effect. $^{12}$

The traditional experimental approach toward the Kondo effect is to measure the resistivity. However, if one considers such measurements in doped metallic clusters in a metallic matrix, then conduction electrons leave and enter the particles, the Kondo cloud extends beyond the nanoparticle, and the distinction between bulk and nanoparticle Kondo effects blurs. In the opposite limit of an insulating or semiconducting matrix, the relative signal by nanoparticles strongly decreases due to the enhanced resistive contrast between particle and matrix.

It is possible to directly measure Fano-Kondo resonances of nanostructures such as atomic contacts, for example, by scanning tunneling microscope, ${ }^{1,2,5}$ but these methods are very complicated, partially due to the involvement of unconfined electron states. Our emphasis is on a different and conceptually very simple approach, namely, to analyze the magnetic susceptibility of magnetic impurities in metallic nanoparticles.

\section{RESISTIVITY}

Experimentally, the Kondo effect is normally associated with the resistance minimum ${ }^{4}$ near the Kondo temperature. The resistivity may, in principle, be determined by embedding the nanoparticles in a matrix and measuring the net resistivity change $\delta \rho$ caused by the Kondo effect (Fig. 1). 


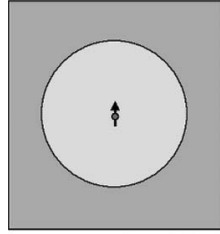

(a)

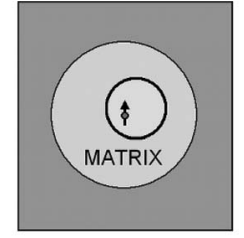

(b)

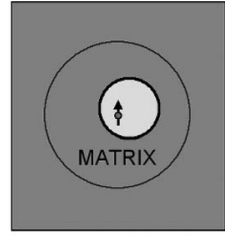

(c)
FIG. 1. Kondo screening clouds around magnetic impurities: (a) bulk solids, (b) nanoparticle in a metallic matrix, and (c) nanoparticle in a nonmetallic matrix. The bright regions are the Kondo screening clouds.

Figure 1 illustrates the involved geometry. Compared to the (a) bulk, there are two limits, namely, (b) embedding in a metallic matrix and (c) embedding in an insulating matrix. In (b), the Kondo cloud (bright) extends deep into the matrix, and the mesoscopic Kondo cloud is no longer well-defined. In (c), the Kondo cloud is confined to the nanoparticle, but the question arises how the insulating character of the matrix affects the nanoparticles' contribution to the resistivity.

A simple approach is to treat the cluster and its surrounding on a continuum level, by using the Bruggeman (or Maxwell-Garnett) approach in analogy to the inverse electric and magnetic susceptibilities of composites. ${ }^{13,14}$ For small volume fractions $f$ of the cluster regions, the effective resistivity obeys

$$
\rho=\rho_{\mathrm{M}}\left(1+3 f \frac{\rho_{\mathrm{P}}-\rho_{\mathrm{M}}}{2 \rho_{\mathrm{P}}+\rho_{\mathrm{M}}}\right),
$$

where $\rho_{\mathrm{P}}$ and $\rho_{\mathrm{M}}$ are the resistivities of matrix and particles, respectively. For small resistivity contrast, where $\rho_{\mathrm{P}} \approx \rho_{\mathrm{M}}$, the effective resistivity is equal to the volume-averaged resistivity and the corresponding Kondo resistivity change $\Delta \rho / \rho=f \delta \rho / \rho_{\mathrm{P}}$ is relatively easy to measure. However, a small resistivity contrast means that there is a strong hybridization between the conduction electrons of matrix and particle. This causes the Kondo screening cloud to extend deep into the matrix, so that the volume fraction $f$ and the change $\delta \rho$ are no longer well-defined. The hybridization problem can be circumvented by using a highly resistive matrix, which confines the Kondo cloud to the particle. Unfortunately, this yields a strong reduction in the relative resistivity change to $\Delta \rho / \rho=3 f \delta \rho / \rho_{\mathrm{M}}$, reaching $\Delta \rho / \rho=0$ for an insulating matrix $\left(\rho_{\mathrm{M}}=\infty\right)$.

\section{SUSCEPTIBILITY}

It is well known that the bulk Kondo effect leads to a strong reduction in the low-temperature magnetic susceptibility $\chi$ (Refs. 15 and 16) and that the drop starts in the vicinity of the Kondo temperature

$$
T_{\mathrm{K}}=W \exp (1 / \kappa) .
$$

Here $W$ is roughly equal to the width of the conduction band and $\kappa=|J| D\left(E_{F}\right)$ is a coupling parameter describing the exchange interaction between impurity spin and conduction electrons. To simplify the comparison with the paramagnetic susceptibility $C / T$, it is convenient to write

$$
\chi=\frac{C}{T}\left[1-F\left(T / T_{\mathrm{K}}\right)\right]
$$

where the function $F\left(T / T_{\mathrm{K}}\right)$ describes how the coupling to the Kondo cloud causes the impurity spin to become "locked." At zero temperature $F=1$, corresponding to complete screening, whereas $T=\infty$ reproduces the paramagnetic limit $(F=0)$.

The transition between the high-temperature and lowtemperature regimes occurs in the vicinity of the Kondo temperature. The high-temperature susceptibility is Curie-like, $\chi=C / T$, but at $T=4 T_{\mathrm{K}}$ the susceptibility is reduced to about $0.5 C / T(F=50 \%)$ and at $T=0.1 T_{\mathrm{K}}$ it reaches about $0.04 C / T$ $(F=96 \%) .{ }^{15}$ The asymptotic high-temperature behavior is ${ }^{15}$

$$
\chi=\frac{C}{T}\left[1-\frac{1}{\ln \left(T / T_{\mathrm{K}}\right)}\right]
$$

or $F=1 / \ln \left(T / T_{\mathrm{K}}\right)$. The same logarithmic expression occurs in the Kondo resistivity ${ }^{8}$

$$
\rho(T) / \rho_{\mathrm{o}}=\text { const. }-\ln \left(T / T_{\mathrm{K}}\right) .
$$

More generally, both the resistivity and the susceptibility are described by $F\left(T / T_{K}\right)=\Phi^{-1}\left[\ln \left(T / T_{\mathrm{K}}\right)\right]$ where $\Phi$ is universal, that is, independent of the details of the metallic host. ${ }^{15}$ The function $\Phi$ depends on $T_{\mathrm{K}}$ but not separately on other quantities, such as the $s-d$ exchange $J$ and the density of states $D\left(E_{F}\right)$.

The logarithmic $\ln \left(T / T_{\mathrm{K}}\right)$ term reflects the sharpness of the Fermi surface. ${ }^{8}$ Treating the interaction $J$ by perturbation theory implies that conduction electrons temporarily occupy states above the Fermi level. The effect is huge at very low temperatures and notoriously difficult to treat by perturbation theory, but the thermal smearing of the Fermi surface means that a typical conduction electron must change its energy by a value of order $k_{\mathrm{B}} T$. Second-order perturbation theory involves a wave-vector integration over inverse energy differences, $1 /\left(E_{\mathbf{k}}-E_{\mathbf{k}^{\prime}}\right)$, and can therefore be used at high temperatures, where it yields the $\ln \left(T / T_{\mathrm{K}}\right)$ term. ${ }^{8,11}$

Nanoparticles do not have a sharp Fermi surface enclosing a continuum of $k$-states but discrete $k$-points in reciprocal space and a gap between the highest occupied and lowest unoccupied states. When $k_{\mathrm{B}} T_{\mathrm{K}}$ becomes smaller than the typical energy spacing $\Delta$ between the $k$-points, then the Kondo effect cannot fully develop, because the conduction electrons need states to be scattered into. This happens for particle radii $R$ smaller than about $W / k_{\mathrm{F}} k_{\mathrm{B}} T_{\mathrm{K}}$. Basically, this length is equal to the Kondo coherence length $\xi \sim v_{\mathrm{F}} / T_{\mathrm{K}}$, which describes the size of the Kondo cloud. Past efforts to experimentally verify the relation between feature size $R$ and Kondo coherence length $\xi$ have been inconclusive. ${ }^{4,6}$ A fundamental reason for these difficulties seems to be the interference by unconfined conduction electrons in the investigated wire and thin-film structures, similar to Fig. 1(b). For example, in thin wires, the wave vectors of the itinerant electrons are discrete in the plane perpendicular to the wire axis but continuous in the direction parallel to the wire. This wave-vector continuum ensures Kondo excitations down to very low temperatures. 


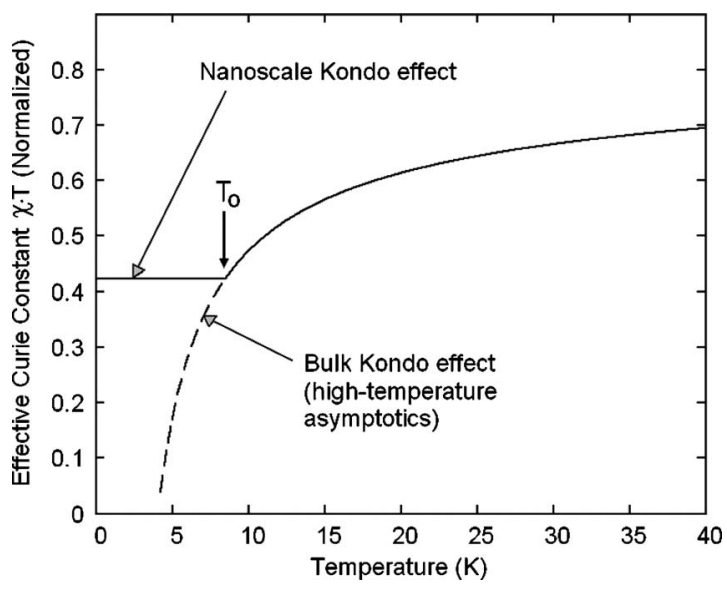

FIG. 2. Kondo susceptibility as a function of temperature (schematic). The figure shows $\chi T=1-F$ for bulk systems (dashed line) and nanoparticles (solid line). The transition temperature $T_{\mathrm{o}}$ decreases with increasing particle size.

Figure 2 compares the Kondo susceptibilities of localized magnetic spins in bulk solids and nanoparticles. For clarity, the figure shows the normalized effective Curie constant $\chi T / C=(1-F)$. The transition temperature $T_{\mathrm{o}}$ is roughly equal to $W / k_{\mathrm{F}} k_{\mathrm{B}} R \sim T_{\mathrm{K}} \xi / R$. Experimentally, the situation may be complicated by the interference from RKKY-like impurity-interaction effects due to the accidental presence of two or more impurities in one nanoparticle. However, since the $\ln \left(T / T_{\mathrm{K}}\right)$ term yields a very sluggish approach to saturation, the horizontal line in Fig. 2 remains significantly below $\chi T / C=1$. In other words, the nanostructuring severely reduces the size of the screening cloud, but there remain sufficient conduction electrons in the nanoparticle to realize a substantial residual Kondo effect.

\section{DISCUSSION AND CONCLUSIONS}

It is instructive to compare the logarithmic approach of Eq. (4) and Fig. 2 with that caused by ordinary AFM interactions. Consider a pair of Heisenberg spins with AFM interactions $J=k_{\mathrm{B}} T_{\mathrm{K}} / 2$, characterized by the eigenfunctions $|\uparrow \uparrow\rangle,|\uparrow \downarrow \pm \downarrow \uparrow\rangle,|\downarrow \downarrow\rangle$, and by the singlet-triplet splitting $\pm J$. The pair's susceptibility is obtained readily as

$$
\chi=\frac{C}{T}\left[\frac{4}{3+\exp \left(T_{\mathrm{K}} / T\right)}\right]
$$

and yields the high-temperature limit $F=T_{\mathrm{K}} / 4 T$. This approach is similar to the asymptotics of the AFM Curie-Weiss law $(F=\theta / T)$ but very different from the logarithmic Kondo expression $F=1 / \ln \left(T / T_{\mathrm{K}}\right)$. It means that nanoparticles vio- late the universality of the bulk Kondo effect and that this violation is most pronounced for the Kondo interaction with a single delocalized orbital. With increasing number of involved $k$-vectors, the signature of the Kondo effect changes and the logarithmic term becomes predominant.

In conclusion, our model analysis shows how the Kondo effect is modified by embedding the impurity spin in a nanoparticle. The resistivity of such clusters is difficult to measure, because the embedding in a metallic matrix yields bulklike Kondo clouds, whereas insulating matrices strongly reduce the signal from the magnetic impurity. However, measurements of the magnetic susceptibility do not suffer from these shortcomings. The Kondo effect persists down to very small particle sizes, but the signature of the effect changes. Below a transition temperature that increases with decreasing particle size, the effective Curie constant reaches a plateau and the temperature dependence of the susceptibility becomes nonuniversal.

\section{ACKNOWLEDGMENT}

This work is supported by NSF-MRSEC, ARO, and NCMN

${ }^{1}$ A.-D. Zhao, Q.-X. Li, L. Chen, H.-J. Xiang, W.-H. Wang, Sh. Pan, B. Wang, X.-D. Xiao, J.-L. Yang, J. G. Hou, and Q.-Sh. Zhu, Science 309, 1542 (2005).

${ }^{2}$ M. R. Calvo, J. Fernández-Rossier, J. J. Palacios, D. Jacob, D. Natelson, and C. Untiedt, Nature (London) 458, 1150 (2009).

${ }^{3} \mathrm{~K}$. Kang, in Condensed Matter Theories, edited by M. P. Das and F. Green (Nova Science Publishers, Commack, 2003), pp. 103-110.

${ }^{4}$ V. Chandrasekhar, P. Santhanam, N. A. Penebre, R. A. Webb, H. Vloeberghs, C. Van Haesendonck, and Y. Bruynseraede, Phys. Rev. Lett. 72, 2053 (1994).

${ }^{5}$ W. B. Thimm, J. Kroha, and J. von Delft, Phys. Rev. Lett. 82, 2143 (1999).

${ }^{6}$ O. Újsághy and A. Zawadowski, J. Phys. Soc. Jpn. 74, 80 (2005).

${ }^{7}$ W. Meissner and B. Voigt, Ann. Phys. 399, 761 (1930).

${ }^{8}$ J. Kondo, Prog. Theor. Phys. 32, 37 (1964).

${ }^{9}$ P. Fulde, Electron Correlations in Molecules and Solids (Springer, Berlin, 1991).

${ }^{10}$ R. Skomski, J. Phys.: Condens. Matter 19, 315202 (2007).

${ }^{11}$ J. M. Ziman, Principles of the Theory of Solids (Cambridge University Press, Cambridge, 1972).

${ }^{12}$ G. Bergmann, Phys. Rev. Lett. 67, 2545 (1991).

${ }^{13}$ D. A. G. Bruggeman, Ann. Phys. 24, 636 (1935).

${ }^{14} \mathrm{R}$. Skomski, Simple Models of Magnetism (Oxford University Press, Oxford, 2008)

${ }^{15}$ H. R. Krishna-Murthy, K. G. Wilson, and J. W. Wilkins, Phys. Rev. Lett. 35, 1101 (1975).

${ }^{16}$ K. D. Schotte and U. Schotte, Phys. Lett. A 55, 38 (1975). 